\title{
The relationship between oral health risk and disease status and age, and the significance for general dental practice funding by capitation
}
IN BRIEF
- Oral health tends to decline with age and can place a great burden on dental practices caring for older age groups.
- An algorithm for recommending capitation fee bands which map these trends is available.
- Capitation payments can be designed to fund care across a broad range of oral health and future disease risk.
- Planners of care funding must subsidise the care of those in greatest need.

\author{
M. Busby, ${ }^{*}$ J. A. Martin, ${ }^{2}$ R. Matthews, ${ }^{3}$ F. J. T. Burke ${ }^{4}$ and I. Chapple ${ }^{5}$
}

\section{VERIFIABLE CPD PAPER}

\begin{abstract}
Aim The aim of this paper was to review the oral health and future disease risk scores compiled in the Denplan Excel/ Previser Patient Assessment (DEPPA) data base by patient age group, and to consider the significance of these outcomes to general practice funding by capitation payments. Methods Between September 2013 and January 2014 7,787 patient assessments were conducted by about 200 dentists from across the UK using DEPPA. A population study was conducted on this data at all life stages. Results The composite Denplan Excel Oral Health Score (OHS) element of DEPPA reduced in a linear fashion with increasing age from a mean value of 85.0 in the 17-24 age group to a mean of 72.6 in patients aged over 75 years. Both periodontal health and tooth health aspects declined with age in an almost linear pattern. DEPPA capitation fee code recommendations followed this trend by advising higher fee codes as patients aged. Conclusions As is the case with general health, these contemporary data suggest that the cost of providing oral health care tends to rise significantly with age. Where capitation is used as a method for funding, these costs either need to be passed onto those patients, or a conscious decision made to subsidise older age groups.
\end{abstract}

\section{BACKGROUND}

Alemayehu and Warner ${ }^{1}$ (2004) estimated that one third of lifetime healthcare costs are incurred by patients of middle age and that half of all lifetime healthcare costs are incurred during the 'senior' years. This growing burden of general healthcare costs as we age is widely recognised and threatens the viability of traditionally funded healthcare systems. As recently as 35 years ago, when many 'senior' citizens were edentulous in the UK (vide infra), and costly dental implants were barely an option, this pattern of general healthcare spending may not have been applicable to oral healthcare. Contemporary evidence now suggests that the age-related pattern of oral health care costs is likely to

\footnotetext{
'Dental Advisor, Denplan and Hon Lecturer in Primary Dental Care University of Birmingham; ${ }^{2}$ Private Practice, Pennsylvania, USA; Chief Science Officer, PreViser Corporation, Washington, USA: ${ }^{3}$ Chief Dental Officer, Denplan Ltd, Winchester; ${ }^{4}$ Professor of Primary Dental Care, Birmingham School of Dentistry; ${ }^{5}$ Professor of Periodontology and Consultant in Restorative Dentistry, Periodontal Research Group and MRC Centre for Immune Regulation, College of Medical and Dental Sciences, University of Birmingham

Correspondence to: Dr Michael Busby

Email:mikeb@denplan.co.uk
}

Online article number E19

Refereed Paper - accepted 2 September 2014

DOI: 10.1038/sj.bdj.2014.1006

${ }^{\circ}$ British Dental Journal 2014; 217: E19 be similar to those of general healthcare.

The Adult Dental Health Survey ${ }^{2}$ (ADHS) conducted in England, Northern Ireland and Wales in 2009 confirmed, as expected, an increasing prevalence of inflammatory periodontal disease with age in the dentate population. The ADHS also confirmed that patients are keeping many more teeth into old age, testimony to the success of preventive dental care strategies of the last two decades. Only 35 years ago, 28\% of the population were edentulous, whereas the figure is now 6\%. Furthermore, it was also reported that patients older than 75 now experience the highest prevalence of dental caries of any age group. The ADHS also confirmed the likely burden of ongoing need for restorative maintenance in older patients; for example ADHS data indicated that only $5 \%$ of the 16-24 age group had a crown compared with more than 55\% of the sample aged over 45 . Generally the ADHS confirms that older patients have fewer sound teeth and more restored teeth.

It was therefore important to ascertain how these general findings might be reflected in patients attending general dental practices in the UK.

In 2013 Busby et al. ${ }^{3}$ reported the development of the Denplan Excel/Previser Patient Assessment (DEPPA) which is an online risk and disease assessment tool for general dental practice. DEPPA reports are based on just over 50 lifestyle, dental history and clinical inputs and comprise the following three elements:

- PreViser $^{\mathrm{TM}}$ risk scores for periodontal disease, caries, non-carious tooth surface loss and oral cancer

- The Denplan Excel Oral Health Score (OHS), which measures oral health status, modified to incorporate PreViser's disease severity measurements for periodontal health and tooth health

- A fee indicator for Denplan Care capitation contracts.

Both the OHS ${ }^{3,4}$ and the PreViser periodontal risk ${ }^{5}$ scoring system have been extensively validated. Risk scoring in DEPPA for caries, tooth wear and oral cancer is based on current expert opinion from the global literature. DEPPA is currently being used by dentists who are part of a voluntary practice accreditation scheme, Denplan Excel. ${ }^{6}$

The data submitted by practices to compile these reports are held centrally in an encrypted and de-personalised form so that only the submitting practice can identify individual patients. However, as reported by Busby et al. ${ }^{7}$ in 2014, the data is held so that reports can be generated in order to produce a national benchmark, audit tables, 


\begin{tabular}{|c|c|}
\hline Severe periodontal disease & $35 \mathrm{pts}$ \\
\hline Moderate periodontal disease & 20pts \\
\hline Mild periodontal disease & 10pts \\
\hline Gingivitis only & $5 p t s$ \\
\hline Healthy & Opts \\
\hline
\end{tabular}

Table 2 Fee code points allocation for future disease risk in each category of caries $(n=5$ max), periodontal disease $(n=5$ max $)$ tooth wear $(n=5$ max) and oral cancer ( $n=5$ max). A maximum of 20 points for future disease risk for dentate patients. Patients with no natural teeth score $\mathbf{0}$ for caries risk, periodontal risk and wear risk

\begin{tabular}{l|l} 
Very high risk & $5 p t s$ \\
\hline
\end{tabular}

\begin{tabular}{|l|l|}
\hline High risk & 4 pts \\
\hline Moderate risk & 3 pts \\
\hline Low risk & 2 pts \\
\hline Very low risk & 1 pt \\
\hline
\end{tabular}

Table 3 Points allocated per tooth for restorative status*

\begin{tabular}{l|l}
\hline Simple restoration $<30 \%$ of coronal surface & $1 p t$ \\
\hline $\begin{array}{l}\text { Complex restoration }>30 \% \text { of coronal } \\
\text { surface }\end{array}$ & $2 p t s$ \\
\hline Root filling & $2 p t s$ \\
\hline Crown post & $2 p t s$ \\
\hline False tooth fixed restoration & $2 p t s$ \\
\hline Removable false tooth & $1 p t$ \\
\hline $\begin{array}{l}\text { *There is no maximum points score for tooth status. The points } \\
\text { from all three tables are totalled and Table } 4 \text { shows the fee band } \\
\text { allocation according to the point's total }\end{array}$
\end{tabular}

and for population analytics. The aim of this paper is to review the oral health status and future risk score data compiled in DEPPA by patient age group and to consider the potential significance of these outcomes to general practice funding by capitation payments, in an aging UK population.

\section{METHODS}

The development and evaluation of the DEPPA oral health and disease risk scoring systems, including diagnostic criteria for the different clinical descriptors has been described previously. ${ }^{3,7}$ Caries and periodontitis diagnoses rely upon clinical and radiographic examination as appropriate. However, Tables 1-4 provide information on the allocation of the points that drive the fee coding within the DEPPA system, as

Table 4 Total points and fee band allocation
\begin{tabular}{|l|l|l|l|l|}
\hline $0-14$ pts & $15-30$ pts & $31-60$ pts & $61-81$ pts & 82 and above \\
\hline A & B & C & D & E \\
\hline
\end{tabular}

\section{Table 5 The number of patients assessed in each age group}

\begin{tabular}{|l|l|l|l|l|l|l}
\hline $17-24$ & $25-34$ & $35-44$ & $45-54$ & $55-64$ & $65-74$ & Over 75 \\
\hline 452 & 703 & 924 & 1730 & 1921 & 1428 & 629 \\
\hline
\end{tabular}

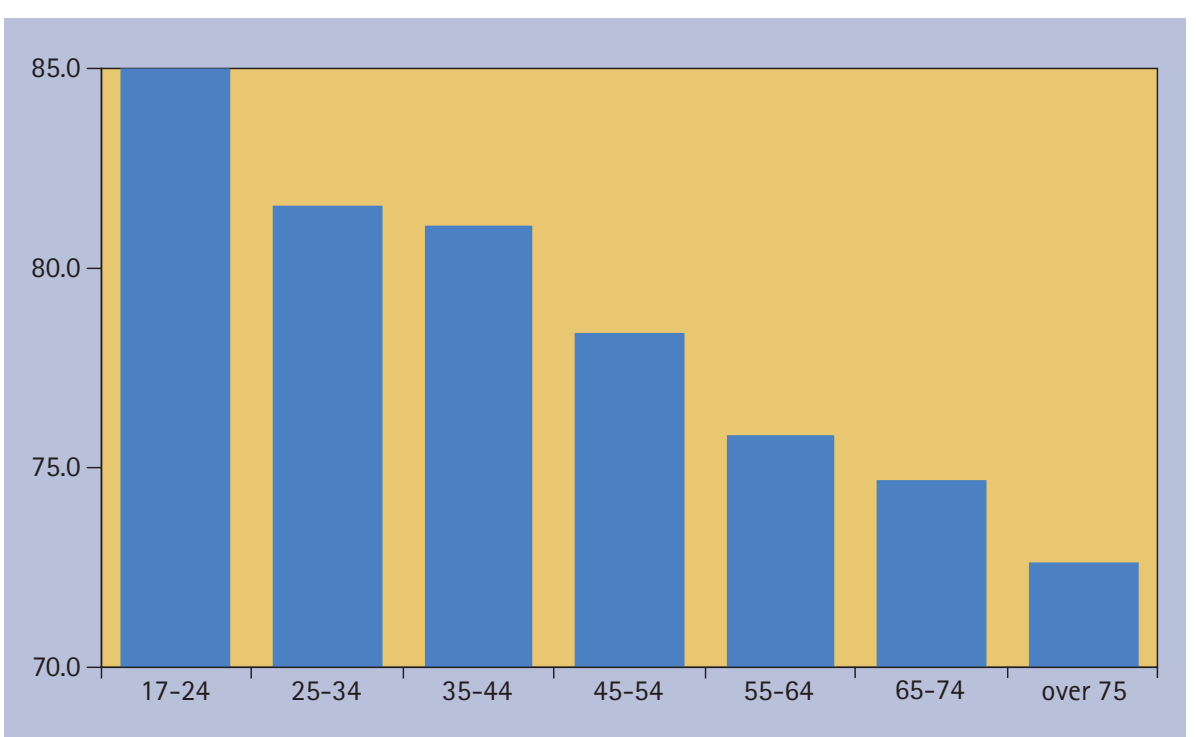

Fig. 1 Mean oral health score by age group

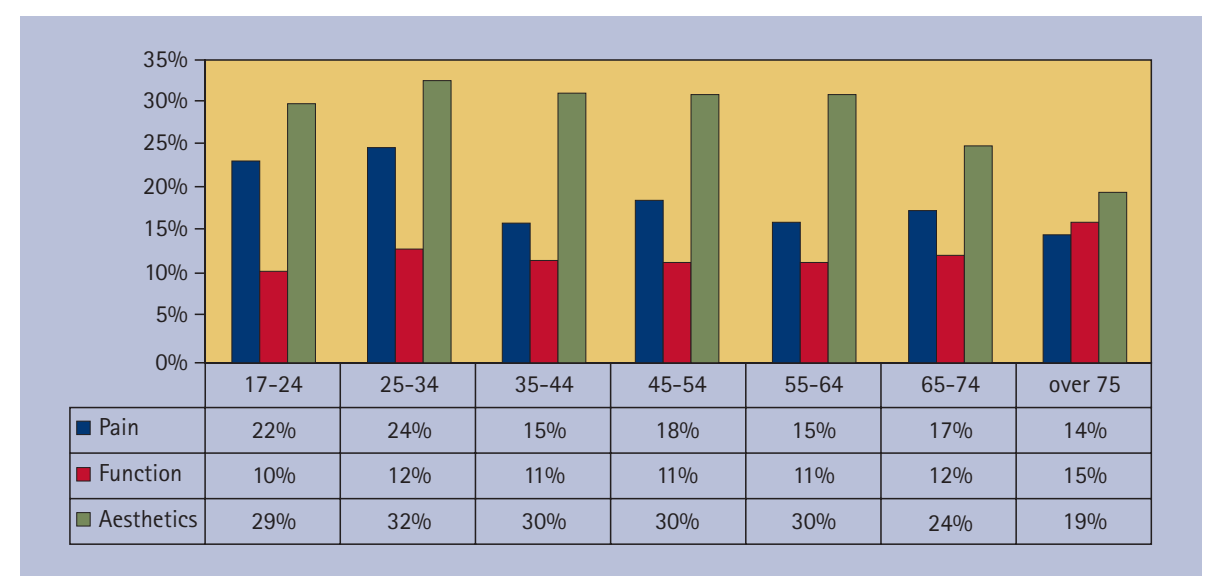

Fig. 2 Percentage reporting any life impact in age groups

this is relevant to the current manuscript. The outcomes of the point's allocation have been previously evaluated in a pilot study of 25 dentists and did not result in large scale changes for patients from the existing manual recording system. ${ }^{7}$ Here we evaluate the fee point scores in relation to age.

Seven thousand, seven hundred and eighty-seven patient assessments were performed between September 2013 and January 2014 by 200 dentists from across the UK using DEPPA's online data entry and submission platform, which has been previously reported. ${ }^{7}$ Reports can be generated at a population as well as an individual practice level and the former was used to conduct a retrospective population study in order to investigate how oral health status and future disease risk vary with age in a group of patients, such as this, attending general dental practices, and how this impacts upon the fee structure within a private capitationbased system.

\section{RESULTS}

Figure 1 reports the average oral health score for seven age groupings recorded at their assessment visit. As previously reported in detail,, 3 the OHS is a composite measurement of oral health taking eight groups of 


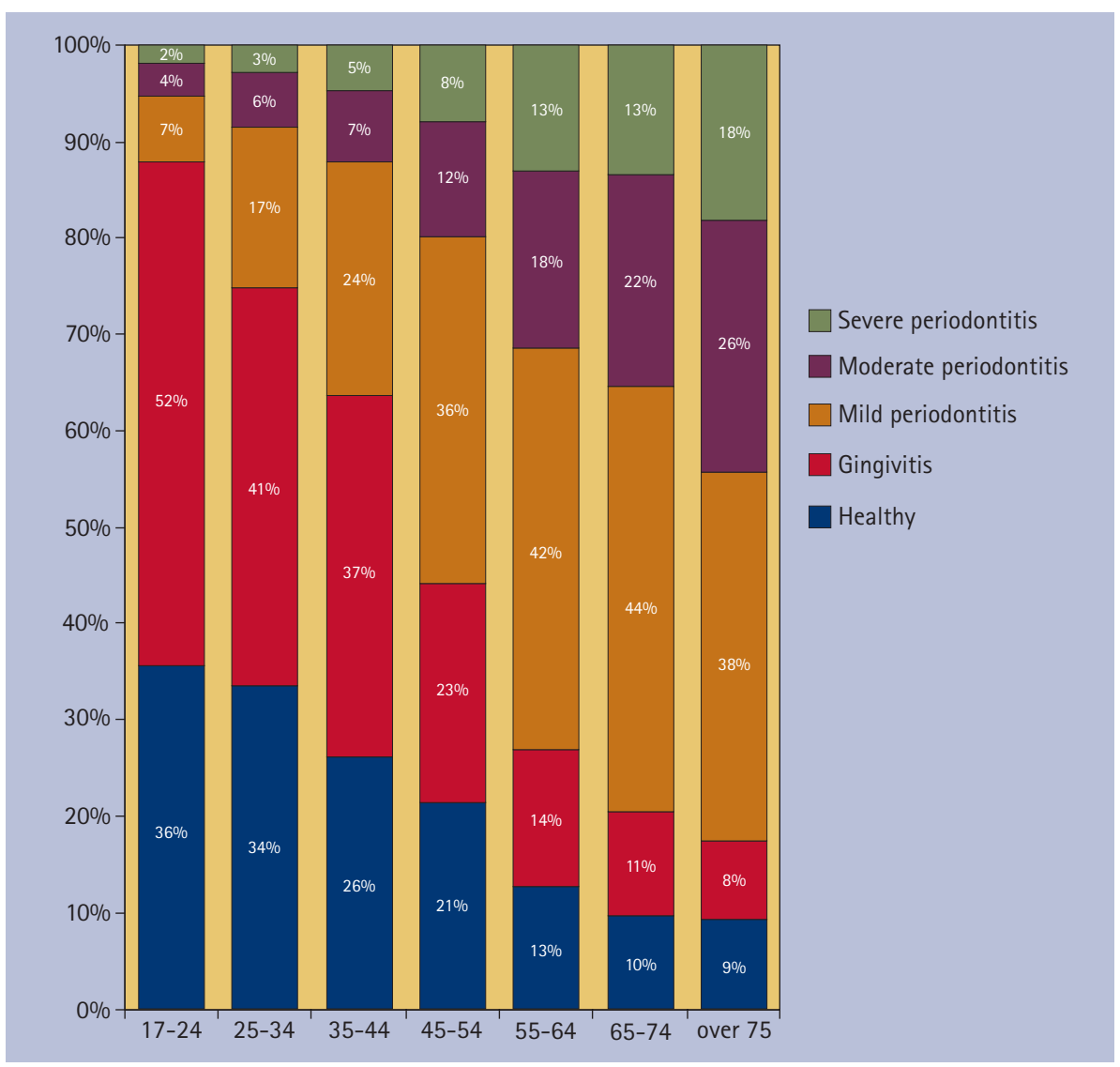

Fig. 3 The percentage of patients in each age group and their periodontal status

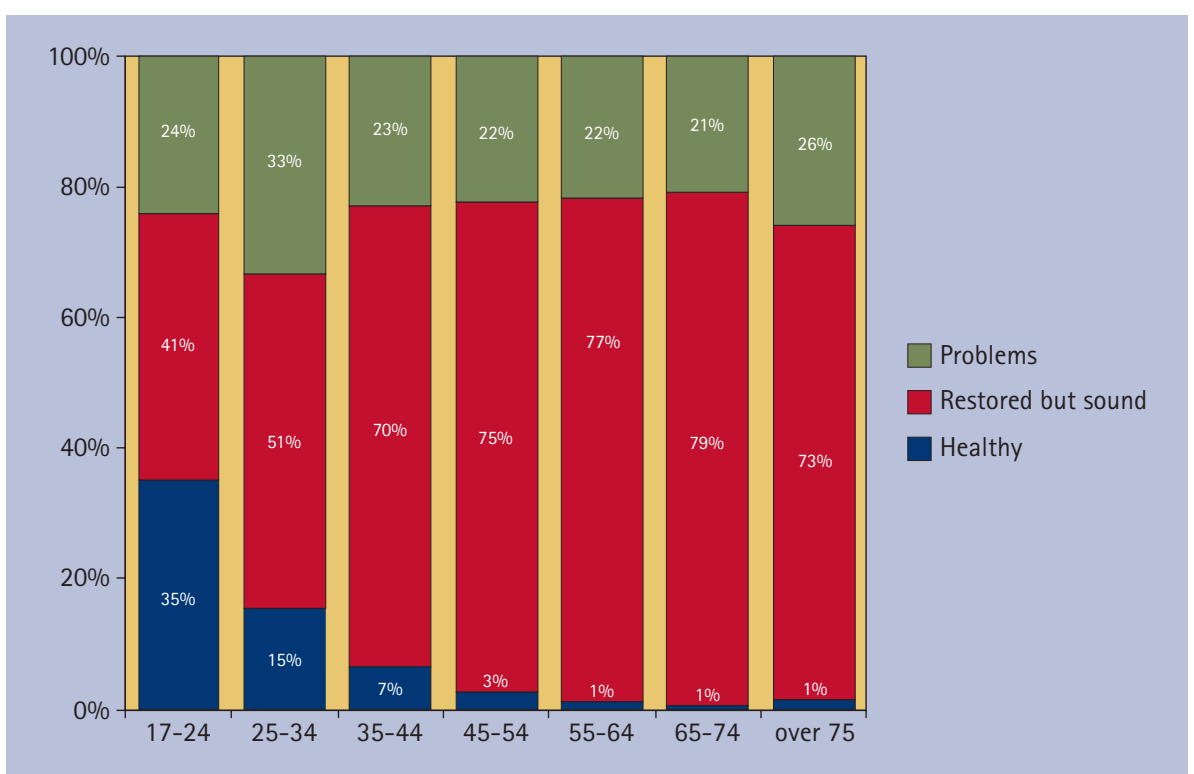

Fig. 4 The percentage of patients in each age group and their tooth health DEPPA grading

factors into account. In the OHS a score of 100 represents perfect oral health. Table 5 reports on the numbers of patients assessed in each age group.

Figure 2 reports specifically on the three patient perception OHS aspects of 'comfort', 'function' and 'appearance'.

Figure 3 reports specifically on the periodontal health aspect of the OHS. Periodontal health is scored in DEPPA as either good health, gingivitis only, mild disease, moderate disease or severe disease. ${ }^{3,7}$ This chart health band in each of the seven age groups.

Figure 5 records the patients graded by the PreViser risk algorithms used in DEPPA as either high or very high risk (scores four and five) for caries and for periodontal disease in the seven age groups.

Figure 6 records the patients graded as high or very high risk (scores four and five) by the PreViser algorithms used in DEPPA for tooth wear and oral cancer.

\section{DISCUSSION}

This population of 7,787 patients is not a random sample of community dwelling adults in the UK, more a convenience sample of Denplan Excel patients, and it is thus not representative of the UK population as a whole. Despite this limitation, it does provide valuable insights into variations in risk and disease profiles by age in primary care dental practice. The participating practices are self-selecting by volunteering for practice accreditation, and further, the dentists in this study are the early adopters of DEPPA. It is probable, due to the nature of the volunteering practices, the majority of these patients are being treated under private capitation contract (Denplan Care ${ }^{6}$ ), and results should therefore be interpreted with this in mind. Nevertheless, given that the current NHS capitation pilot schemes are driven by risk and disease scores, the reported data and the age-related patterns within this population do have relevance to the broader NHS.

An overview of the data in Figures 3 and 4 reveals that the recorded pattern of disease experience relative to age group tends to follow the pattern observed in the ADHS. In respect of periodontal disease (Fig. 3) the prevalence of disease (mild, moderate and severe) appears to increase linearly $\left(R^{2}=0.97 ; p<0.0001\right)$ with age from age $25-74$, which is also the case for severity of disease $\left(R^{2}=0.87 ; p<0.0001\right)$. In respect of caries (Fig. 4) the prevalence of restored or carious teeth (not healthy) increases linearly $\left(\mathrm{R}^{2}=0.81 ; \mathrm{p}<0.0001\right)$ with age from 17-54 years and the pattern of caries also follows the age-related pattern of the ADHS, with peaks in caries (or defective restorations) in the oldest age group and the 25-35 year age groups. These peaks are however significantly lower in this group of patients compared to the general population investigated in the ADHS, as might be expected because of the nature of their selection for this study, and the preventive philosophy of the practices involved.

Figure 1 demonstrates, through the OHS, how oral health deteriorates linearly $\left(\mathrm{R}^{2}=-0.83 ; \mathrm{p}<0.0001\right)$ with age. This phenomenon is mostly due to declining periodontal health because periodontal health contributes as $24 \%$ of the OHS. 
Despite the declining 'clinically assessed' oral health status of this population, the two oldest age groups appear to experience fewer key 'life impacts' than younger age groups. These reported life impacts also account for $24 \%$ of the total of the OHS. Figure 2 demonstrates that just under one third of patients report some concerns about their dental appearance in the youngest five age groups. However after the age of 65 this percentage begins to fall, with less than one fifth of the over 75's expressing any concerns about their dental appearance.

There is a peak in the 25-34 age group in reporting dental discomfort. It could be speculated that, as this mirrors the caries prevalence peak, much of this reported discomfort may be a consequence of these lesions. However this age group also presents the highest percentage of patients at high and very high risk from tooth wear (Fig. 6). This may be because of reported diets that are high in acid and therefore tooth sensitivity might also be contributing. Alternatively, McArdle and Renton ${ }^{8}$ concluded in 2012 that following NICE guidance on the removal of wisdom teeth in the United Kingdom in 2000 , the mean age for extraction of these teeth was now 32 rather than 25, as was the case before the NICE guidance. The total number of procedures was now similar to the pre-2000 period. Therefore, patients in this age group may also be experiencing notably more life impacts caused by wisdom teeth, as their extraction seems to have simply been delayed by the NICE guidance. Unfortunately, details of the specific reasons for the reported negative life impacts are not recorded and therefore this observation is speculative.

Figures 5 and 6 report patients scored by the PreViser algorithms in DEPPA as high or very high risk of future disease for four dental conditions. Taking each condition in turn:

- The caries risk scores, as one might expect, clearly mirror the peaks of caries prevalence

- The high risk peak of future periodontal disease is recorded in patients in their middle years. Reflecting on this phenomenon will reveal the logic of this strongly validated scoring, because it is predicting which patients will develop severe disease by their later years unless risk factors are reduced at a younger rather than an older age.

- Increasing age is a significant risk factor for oral cancer and this is clearly reflected in these data

- There is a peak in the risk of developing non-carious tooth surface loss in the 25-34 age group. This is probably as

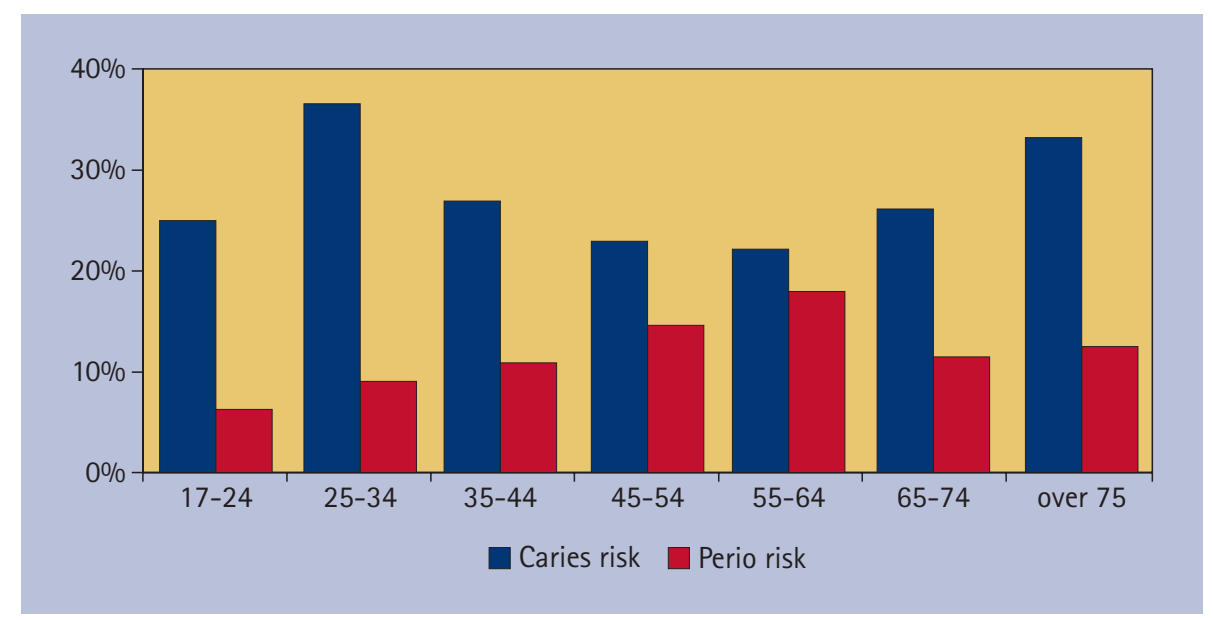

Fig. 5 Percentage high or very high risk for caries and periodontal disease in age groups

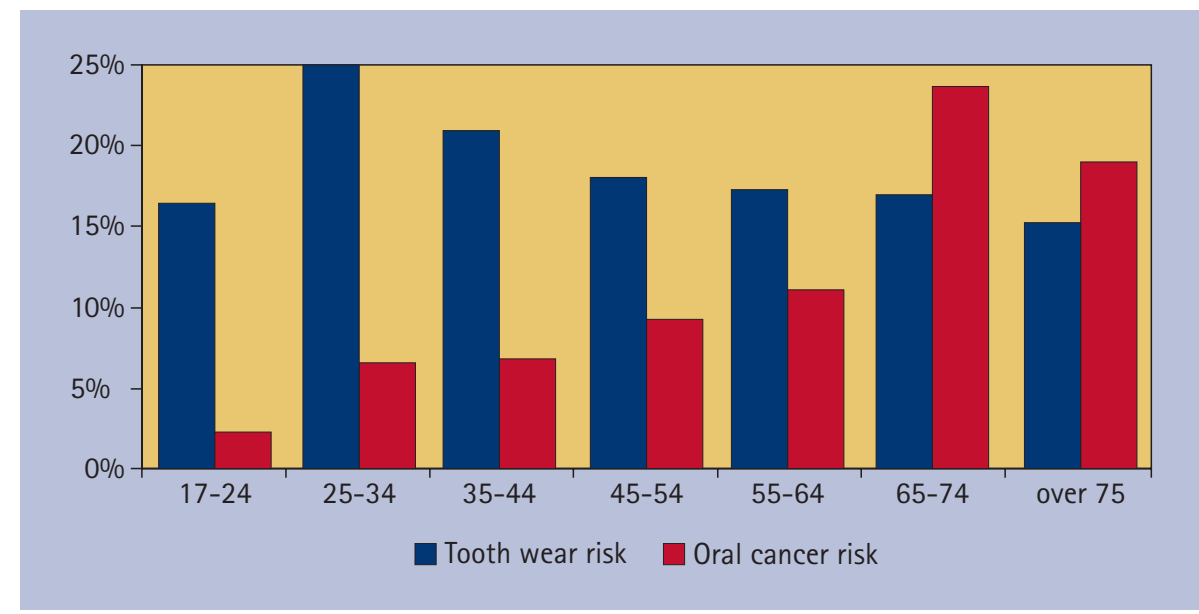

Fig. 6 Percentage of patients high or very high risk of tooth wear or oral cancer

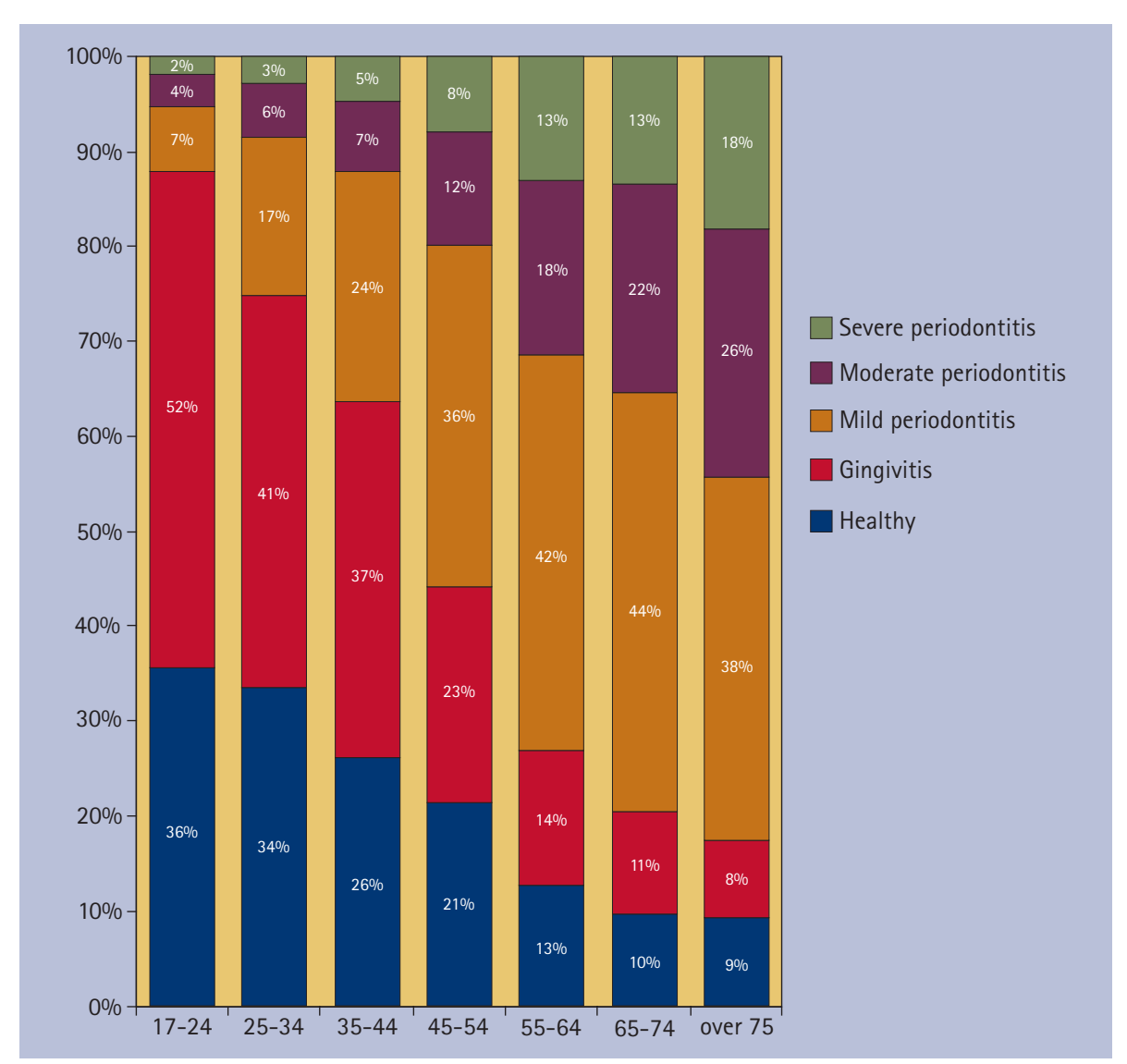

Fig. 7 DEPPA capitation fee guidance (codes A-E) in age groups 
a result of a higher reported intake of acidic food/drinks.

Figure 4 demonstrates how few patients currently make it to their senior years, without having teeth restored or experiencing caries. This illustrates how the restoration maintenance burden on dental practices continues to grow as patient's age, a finding mirrored by the ADHS data.

In a group of patients attending a general dental practice such as this study population, it would appear that the main practice workload would come from the following activities:

- Ongoing patient assessment and general preventive advice at a frequency and intensity appropriate to patients' oral health status and future disease risk for all conditions

- Ongoing periodontal health care at a frequency and intensity guided by periodontal health status, future risk of periodontitis and any life impacts caused by the disease

- Ongoing tooth health care relating to: caries activity and future risk; noncarious tooth surface loss status and future risk; the maintenance needs of the occlusion, existing restorations; and any life impacts caused by any of these.

A review of the findings in this paper and those of the ADHS suggests that, generally, with some exceptions, the oral health needs of general dental practice patients increase as patient's age.

The authors estimate that around 2.5 million patients receive care in the UK private dental sector through self-payment of capitation fees. It has been widely recognised in the private sector that capitation fees should reflect the likely practice workload for a given group of patients.

As part of DEPPA a capitation fee guidance code is generated by the algorithms from inputs on existing oral health status (including restorative status) and the future disease risk scoring. Tables 2-5 illustrate how a points system is used by the DEPPA algorithms to place patients in each category. The fee codes (A,B, C, D and E) are designed to allow practices to price the capitation care costs for their patients at an appropriate level to allocate sufficient time for each patient care group, with A being the lowest monthly fee band and E the highest. The fee level assigned to each fee category is a decision for each individual dentist to make, based on their practice circumstances. The decision on the appropriate fee category for each patient is also at the discretion of each dentist. When structured in this way, capitation fees can be an appropriate way of funding a preventive approach to care for patients in any state of oral health. Figure 7 shows how this fee advice through the DEPPA reports is recommending a trend towards higher fees as the patient ages, for the patients in this study population. Pricing private capitation fees in this manner means that patients requiring the most resources to support their care pay the highest fees. Flat capitation fees would result in those in very good oral health subsidising the care of those in poor oral health.

The Steele Review of NHS dental services in England ${ }^{9}$ (2009) supported a significant role for capitation funding in the future for these services and made the following comments in relation to some of the issues discussed above:

- 'We recommend that the basic value of the capitation payment should be nationally determined, but that it is weighted in a predictable way to take account of practice profile'

Because many adult patients are responsible for patient charges in respect of NHS dental care in England, Steele et al. state that charges:

- Should be simple (their research concluded that up to ten bands would be acceptable)
- Should be fair (they stated that the charge should broadly reflect the cost of providing the service)

- Should provide incentives for patients to embrace good self-care.

These ideas are currently being piloted in England, at the time of writing, and it will be interesting to see how any new payment system in the NHS copes with the age-related resource challenges discussed in this paper.

\section{CONCLUSION}

As is the case with general health, these contemporary data suggest that the cost of providing oral health care tends to rise significantly with age. Where capitation is used as a method for funding, these costs either need to be passed onto those patients, or a conscious decision made to subsidise older age groups.

1. Alemayehu B, Warner K. The lifetime distribution of healthcare costs. Health Serv Res 2004; 39: 627-642.

2. The Health and Social Care Information Centre. Adult dental health survey. 2009. Online information available at http://www.hscic.gov.uk/ pubs/dentalsurveyfullreport09 (accessed October 2014).

3. Busby M, Matthews R, Chapple E, Chapple I. Nove online integrated oral health and risk assessment tool: development and practitioners' evaluation. $\mathrm{Br}$ Dent J 2013; 215: 115-120.

4. Burke F J T, Busby M, McHugh S, Delagy S, Mullins A, Matthews R. Evaluation of an oral health scoring system by dentists in general practice. Br Dent J 2003; 194: 214-218.

5. Page R C, Martin J, Krall E A, Mancl L, Garcia R. Longitudinal validation of a periodontal risk calculator. J Clin Periodontol 2003; 30: 819-827.

6. Denplan. Denplan Excel Accreditation and DEPPA.

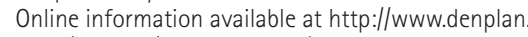
co.uk/dentists/denplan-excel (accessed October 2014)

7. Busby M, Matthews R, Chapple E, Chapple I. Continuous development of an oral health score for oral health surveys and clinical audit. Br Dent J 2014; 216: E20.

8. McArdle L, Renton T (2012). The effects of NICE guidance on the management of third molar teeth. BrDent J 213; 213: E8.

9. Steele J, Rooney E, Clarke J, Wilson T. NHS dental services in England. 2009. NHS England. Online information available at http://www.sigwales.org/ wp-content/uploads/dh_101180.pdf (accessed October 2014). 